\title{
Accuracy Issues for Parameter Estimation of Field Oriented Induction Machine Drives
}

\author{
Diane E. Borgard, Gustaf Olsson, and Robert D. Lorenz, Senior Member, IEEE
}

\begin{abstract}
This paper will attempt to resolve a major area of concern for self-commissioning of induction machine drives: easily implemented methods capable of accurate parameter estimation. Most of the self-commissioning techniques are based on some form of statistical analysis. Classical statistical techniques such as ARMA ( $z$-transform) methods using sampled line voltages, phase currents, and rotor velocity can easily produce large ( $2 \times$ or more) parameter errors. The first principles causing the errors and the methods of reducing those effects to tolerable levels is the focus of this paper.
\end{abstract}

\section{INTRODUCTION}

$\mathbf{P}$ ARAMETER estimation on any system, such as an induction machine, is necessary for many reasons, including proper tuning for field oriented induction machine drives. But whether it is simply for diagnosis or for adaptive (selftuning) control, the physical parameters of the system should be accurately estimated.

Time series analysis, ARMA ( $z$-transform) estimation using least squared error coefficient fitting, has been used for decades to numerically identify system models in the discrete time domain [1]-[3]. With input and output signals sampled at equal time intervals and a linear model structure relating the two sets of signals, coefficients of the numerical model can be found using this technique. From these coefficients, the physical parameters of the system can be calculated. Unfortunately, little attention has been paid to the accuracy of the estimated physical parameters resulting from this identification procedure, especially as applied to induction machine drives [4]-[6]. This is true despite the fact that the tuning of indirect field orientation controllers for induction machine drives uses the physical parameters, not ARMA coefficients.

Very significant accuracy problems can arise when using time series analysis. These problems relate directly to the interaction of finite resolution analog-to-digital converters with the sample rate. Furthermore, these problems are compounded by the wide span of time constants in the machine's electrical, magnetic, and mechanical models. This paper will provide

Paper IPCSD 95-26, approved by the Industrial Drives Committee of the IEEE Industry Applications Society for presentation at the 1994 IEEE Industry Applications Society Annual Meeting, Denver, CO, October 2-7. This work received support from the Wisconsin Electric Machines and Power Electronics Consortium (WEMPEC) of the University of Wisconsin-Madison. Manuscript released for publication March 10, 1995.

D. E. Borgard was with the Department of Electrical and Computer Engineering, University of Wisconsin, Madison, WI 53706-1691 USA. She is currently at St. Charles, MO 63304 USA.

R. D. Lorenz is with the Department of Electrical and Computer Engineering, University of Wisconsin, Madison, WI 53706-1691 USA.

G. Olsson is with the Department of Industrial Electrical Engineering and Automation, Lund Institute of Technology, S-221 00 Lund, Sweden.

IEEE Log Number 9411537. a meaningful explanation of these error contributors and demonstrate techniques to reduce their effects.

\section{SAMPLE RATE SELECTION}

To apply statistical methods for parameter estimation on a field oriented induction machine, the accessible and commonly measured variables, i.e., voltage, current, and velocity, must be sampled and used to estimate a model. The requirements for a valid identification include, but are not limited to: a linear equation model used for estimation, sufficient excitation of the interesting dynamics, anti-alias filtering, and an appropriate sample rate. Of these requirements, the effect of the sample rate is the most misunderstood. A common misconception is that the best identification results can be obtained by sampling as fast as the hardware will allow. Such high sample rates actually cause errors and are therefore unsuitable for time series analysis. This section will explain this aspect of parameter estimation and demonstrate the problems caused by high sample rates.

It is widely known that the sample rate must be fast enough to avoid aliasing. Though less well known, it is demonstrated in [7] that a sample rate that is very fast relative to the system's dynamics can also cause inaccuracies. Furthermore, a high sample rate coupled with the quantization errors inevitably resulting from the finite resolution of the analog-to-digital converters can ruin an otherwise exceptional identification experiment. To understand this effect, the relationships between the continuous time domain and the discrete time domain must be understood.

\section{A. Discrete and Continuous Time Domains}

A stable root in the continuous time domain can fall anywhere in the left half of the $s$-plane. This infinite region corresponds to a unit circle in the $z$-plane. Because an infinite area is condensed into the finite and small area of a unit circle, there are regions of the $z$-plane where the discrete time root is relatively insensitive to the continuous time root. These regions of the discrete time domain should be avoided. Given fixed dynamics, the only way to avoid these regions is with a proper selection of the sample rate.

Fig. 1 demonstrates this effect by overlaying the $z$-domain equivalent of a $100-\mathrm{Hz}$ pole and a $200-\mathrm{Hz}$ pole as a function of sample rate. These two poles have distinct $z$-domain values for a range of sample rates. However, at both relatively slow and relatively fast sample rates, the two $z$-domain roots are barely distinguishable from each other. This lack of separation in the $z$-domain roots has a crucial effect on the identification 


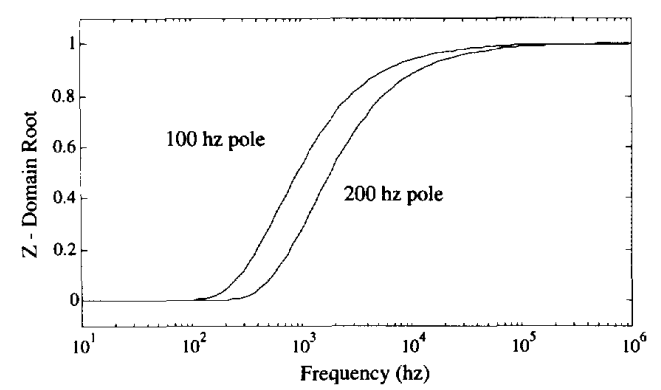

Fig. 1. $z$-domain root versus sample rate for a 100 - and a $200-\mathrm{Hz}$ pole.

problem. Because the continuous time roots and physical parameters are determined from the estimated discrete time model, the $z$-domain roots must be estimated precisely and accurately enough to determine the location of the continuous time roots.

In order to accurately distinguish between $z$-domain roots at sample rates which are fast relative to the speed of the pole, the root must be estimated with high precision. The faster the sample rate relative to the dynamics, the greater the precision necessary to accurately determine the continuous time root. However, at fast sample rates, the high $z$-domain precision necessary to accurately identify the physical parameters is not always attainable. The ability to precisely identify the discrete time roots depends upon the resolution with which the input and output signals can be measured, and on the accuracy with which they represent the modeled dynamics.

\section{B. The Effect of Limited Resolution and Accuracy}

Ideally, the exact values of a continuous time root and a discrete time root are related only by the sample rate. If the discrete time roots can be accurately estimated to an arbitrarily large number of digits, the physical parameters can be accurately estimated at any sample rate. However, limitations on the resolution and accuracy of the estimated discrete time roots are inevitable for practical systems.

At high sample rates, the discrete time roots must be estimated accurately and precisely enough that the pertinent information about the continuous time root, found several digits beyond the decimal point, can be extracted. However, with the commonly used $8-$ and $12-\mathrm{b}$ converters, the resolution is too limited to assure estimation accuracy beyond a few decimal places. As a result, the precise location of the continuous time root cannot be determined from data sampled more than two orders of magnitude faster than the dynamics.

For example, suppose an attempt is made to identify a $100-\mathrm{Hz}$ root. If the discrete time root were estimated very precisely, to within \pm 0.001 , the continuous time root may still be estimated with excessive inaccuracy if an inappropriate sample rate is chosen. Fig. 2 shows this effect, where the only error is a fixed error of \pm 0.001 .

The region between the two lines represents the uncertainty of the estimation results. At sample rates more than two orders of magnitude faster than the dynamics, the errors in the estimated continuous time root increase exponentially to intolerable levels.

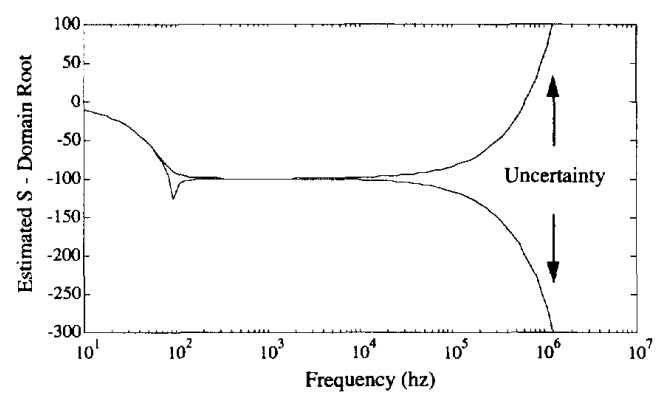

Fig. 2. Continuous time estimate of a $100-\mathrm{Hz}$ root if the discrete time root is estimated with an uncertainty of \pm 0.001 .

The estimated continuous time root is least sensitive to these quantization errors at sample rates just slightly faster than the physical root. Clearly, sampling as fast as the hardware allows is not appropriate. To reduce the effect of limited resolution, an analog-to-digital converter with a "fine" resolution should be chosen. The better the resolution, the less the effect of quantization errors on the estimation results. Furthermore, the sampled signals should be appropriately scaled such that the converter's range is optimally used. Not using the full range of the converter is equivalent to optimally using a converter with a coarser resolution.

Besides the limitations imposed by finite resolution converters, there are many sources of error that effect the accuracy of the estimated parameters. For example, the input and output signals are often noisy. In common induction machine drives, the PWM switching causes the line-to-line voltage and line current signals to contain noise unrelated to the dynamics. Furthermore, any unmodeled or undermodeled dynamics will cause some errors in the estimated discrete time model.

Similar to the effect of limited resolution, errors in the discrete time domain have a greater impact on the estimated continuous time parameters when an inappropriate sample rate is selected. At relatively low and high sample rates, a small error in the estimated value of a discrete time root (the initial estimate from which other values are calculated) causes a significant error in the estimated continuous time root. The estimated continuous time root is least sensitive to errors in the estimated discrete root at sample rates slightly faster than the continuous time root.

There are many possible sources of error in the identification process, and it is important to reduce those errors at the source, when possible. However, such attempts at reducing errors are invalidated once an inappropriate sample rate is chosen. A moderate sample rate cannot replace an intelligent identification experiment. A sample rate too fast, however, can ruin even the best experiment.

\section{Separated Dynamics}

It has been demonstrated that the accuracy of the identification can be improved by sampling at a moderate rate. However, when a system has multiple time constants with vastly different dynamics, it is impossible to accurately capture all the dynamics with one sample rate. Such is the case with the induction machine which has transient electrical dynamics which are often more than an order of magnitude faster 


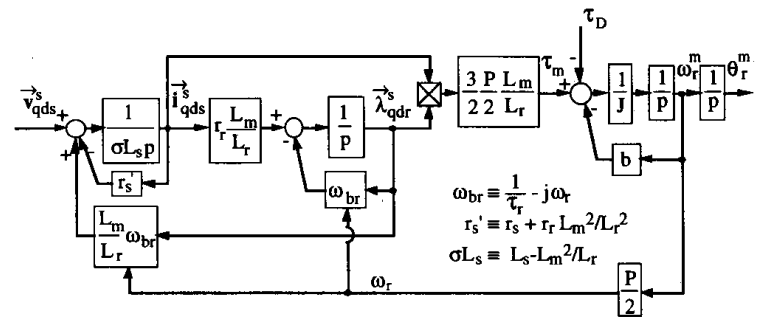

Fig. 3. Stationary frame induction machine model including load.

than the magnetizing flux dynamics, which in turn are often more than an order of magnitude faster than the mechanical load dynamics. Sampling sufficiently fast to capture transient electrical dynamics forces the magnetic and mechanical dynamics to appear as if they were pure integrators. Conversely, sampling sufficiently slow to capture mechanical or magnetic dynamics forces the transient electrical dynamics to be estimated as nearly zero valued time constants (deadbeat sampled response). The electrical, magnetizing, and mechanical dynamics are often so different in time scale that accurate physical parameter estimates cannot be achieved with one sample rate alone. Therefore, using multi-rate sampling, in which the signals are sampled at various frequencies around each anticipated break frequency, the electrical, magnetic, and mechanical dynamics can all be better represented. This results in substantially more accurate physical parameter estimates.

The benefits of multi-rate sampling can be improved by further separating the dynamics in the models and in the identification experiment. A different experiment can then be used to identify physical parameters from each subsystem. The separation of the induction machine model into its dynamic subsystems is explained in the following section.

\section{Forming AN OPERATING POINT MODEL}

Because the least squares algorithm estimates the numerical coefficients of a linear equation model, the inherent nonlinear features of the induction machine must be properly considered for the most accurate estimation. The following (1)-(4) and Fig. 3 show the nature of the nonlinear features of the induction machine represented in the stationary reference frame [8]. (Note: $p$ denotes a derivative operator and $\chi$ denotes a vector cross product)

$$
\begin{aligned}
p \overrightarrow{\mathbf{i}}_{q d s}^{s} & =\frac{1}{\sigma L_{s}}\left(\overrightarrow{\mathbf{v}}_{q d s}^{s}-r_{s}^{\prime} \overrightarrow{\mathbf{i}}_{q d s}^{s}+\frac{L_{m}}{L_{r}} \omega_{b r} \vec{\lambda}_{q d r}^{s}\right) \\
p \vec{\lambda}_{q d r}^{s} & =\frac{1}{\tau_{r}} L_{m} \overrightarrow{\mathbf{i}}_{q d s}^{s}-\omega_{b r} \vec{\lambda}_{q d r}^{s} \\
\tau_{m} & =\frac{3}{2} \frac{P}{2} \frac{L_{m}}{L_{r}} \overrightarrow{\mathbf{i}}_{q d s}^{s} \chi \vec{\lambda}_{q d r}^{s} \\
p \omega_{r}^{m} & =\frac{1}{J}\left[\tau_{m}-b \omega_{r}^{m}-\tau_{D}\right]
\end{aligned}
$$

where $p$ denotes a derivative operator.

The induction machine model is seen to be nonlinear due to the products of variables (flux and speed for induced voltages, and flux and current for torque). In addition, magnetic nonlinearities (main flux and leakage path saturation) cause the physical parameters to vary with operating conditions.

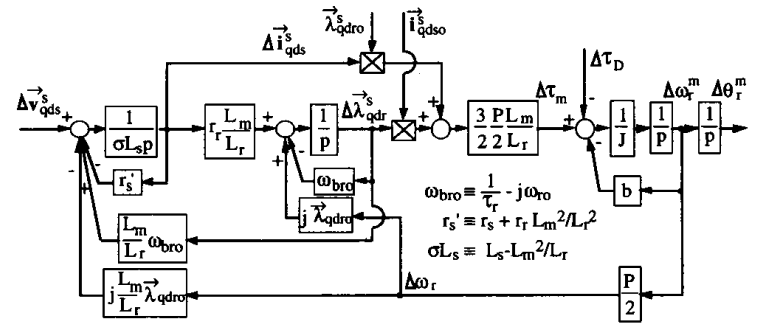

Fig. 4. Stationary frame induction machine operating point model.

From this nonlinear model it is possible to form a statistical parameter estimation approach based on the assumption that speed is a slowly changing variable relative to current and flux. One such model assumes a constant speed [5] and [9]. This assumption allows a second order system differential equation model which is based on derivatives of the current and voltage variables. However, such approaches are severely limited by the need to take derivatives of signals which are already very noisy, i.e., the first derivative of the PWM voltages, and the first and second derivatives of the stator currents.

An alternative approach which does not require derivatives is to form an operating point, or small signal model which allows speed to vary about an operating point. Such a model, represented in the stationary reference frame, is shown in Fig. 4.

For use with field orientation, where the commands are referenced in the synchronous reference frame, these models can be transformed to a reference frame that rotates with the stator operating point flux. The transformation used to convert the stationary frame to the corresponding synchronous frame is shown in (5).

$$
\left\{\begin{array}{l}
f_{q s}^{e} \\
f_{d s}^{e}
\end{array}\right\}=\left[\begin{array}{rr}
\cos \left(\omega_{e} t\right) & -\sin \left(\omega_{e} t\right) \\
\sin \left(\omega_{e} t\right) & \cos \left(\omega_{e} t\right)
\end{array}\right]\left\{\begin{array}{l}
f_{q s}^{s} \\
f_{d s}^{s}
\end{array}\right\} .
$$

The resulting synchronous frame operating point model is described by the following equations and shown in Fig. 5:

$$
\begin{aligned}
p \Delta \overrightarrow{\mathbf{i}}_{q d s}^{e}= & \frac{1}{\sigma L_{s}}\left(\Delta \overrightarrow{\mathbf{v}}_{q d s}^{s}-\left(r_{s}^{\prime}+j \omega_{e} \sigma L_{s}\right) \Delta \overrightarrow{\mathbf{i}}_{q d s}^{e}\right. \\
& \left.+\frac{L_{m}}{L_{r}}\left(\omega_{b r o} \Delta \overrightarrow{\mathbf{i}}_{q d r}^{e}-j \overrightarrow{\mathbf{l}}_{q d r o}^{e} \Delta \omega_{r}\right)\right) \\
p \Delta \vec{\lambda}_{q d r}^{e}= & \frac{L_{m}}{\tau_{r}} \Delta \overrightarrow{\mathbf{i}}_{q d s}^{e}-\left(\omega_{b r}+j \omega_{e}\right) \Delta \vec{\lambda}_{q d r}^{e}+j \vec{\lambda}_{q d r o}^{e} \Delta \omega_{r} \\
\Delta \tau_{m}= & \frac{3}{2} \frac{P}{2} \frac{L_{m}}{L_{r}}\left(\Delta \overrightarrow{\mathbf{i}}_{q d s}^{r} \chi \vec{\lambda}_{q d r o}^{e}+\overrightarrow{\mathbf{i}}_{q d s o}^{e} \chi \Delta \vec{\lambda}_{q d r}^{e}\right) \\
p \Delta \omega_{r}^{m}= & \frac{1}{J}\left[\Delta \tau_{m}-b \Delta \omega_{r}^{m}-\Delta \tau_{D}\right]
\end{aligned}
$$

This fifth order model of the induction machine contains three separate dynamic subsystems: electrical, magnetic, and mechanical. As presented in the previous section, these dynamics cannot all be accurately identified with one sample rate. By separating the model into its subsystems and by selecting an appropriate sample rate for each subsystem, all the machine dynamic parameters can be more accurately identified. This paper focuses on the electrical and magnetic subsystems. 


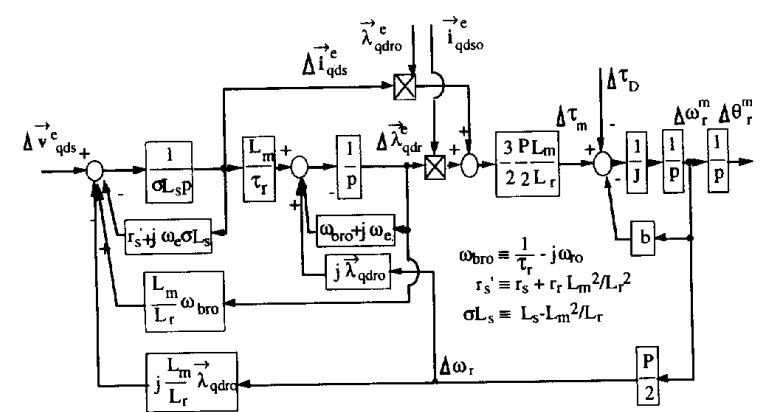

Fig. 5. Synchronous frame induction machine operating point model.

By assuming that the flux and speed remain constant in the time frame of the electrical dynamics, the electrical subsystem can be derived. The resulting transfer function between the current and voltage is

$$
\frac{\Delta \overrightarrow{\mathbf{i}}_{q d s}^{e}}{\Delta \overrightarrow{\mathbf{v}}_{q d s}^{e}}=\frac{\frac{1}{\sigma L_{s}}}{p+\frac{r_{s}^{\prime}}{\sigma L_{s}}+j \omega_{e}} .
$$

From this model, the stator transient time constant, $\frac{\sigma L_{s}}{r^{\prime}}$, can be calculated from the real component of the pole. In addition, the specific values of the stator transient inductance, $\sigma L_{s}$, and the stator transient resistance, $r_{s}^{\prime}$, can be calculated if the static gain is identified. But, with time series analysis, it is more difficult to obtain a reliable estimate for the static gain than for the dynamic pole. A high signal-to-noise ratio can generally improve the accuracy of the estimated gain. However, because small signal analysis is used to model the nonlinear behavior of the induction machine, such noise causes the inputs and states to deviate too much from the operating point values. Increasing the signal-to-noise ratio, therefore, can actually cause the accuracy of the estimation results to deteriorate. As a result, a separate step response test is used to determine the static gain.

Next, by assuming that the current reaches its steady-state value instantaneously and the speed remains constant relative to the magnetic dynamics, the magnetic subsystem can be derived. The resulting model is

$$
\frac{\Delta \overrightarrow{\mathbf{i}}_{q d s}^{e}}{\Delta \overrightarrow{\mathbf{v}}_{q d s}^{e}}=\frac{\frac{p+\omega_{b r a}+j \omega_{e}}{r_{s}^{\prime}+j \sigma L_{s} \omega_{e}}}{p+\left(\omega_{b r o}+j \omega_{e}\right)-\frac{L_{m}^{2} \omega_{b r^{\prime}}}{L_{r} \tau_{r}\left(r_{s}^{\prime}+j \sigma L_{s} \omega_{e}\right)}} .
$$

The rotor time constant can be determined from the real component of the zero of this model.

Finally, the motor inertia and damping can be determined from the mechanical load subsystem. While not specifically discussed, these parameters can be determined by following the same techniques outlined in this paper.

These linear coefficient models accurately represent the incremental properties of the induction machine system about operating points. Thus, time series analysis can be performed on the induction machine using these models. A sufficient range of operation of the machine must be included to assure that all reasonable operating points have been estimated. If this is performed properly, then it is possible to estimate operating point physical parameters from the operating point time series model coefficients.

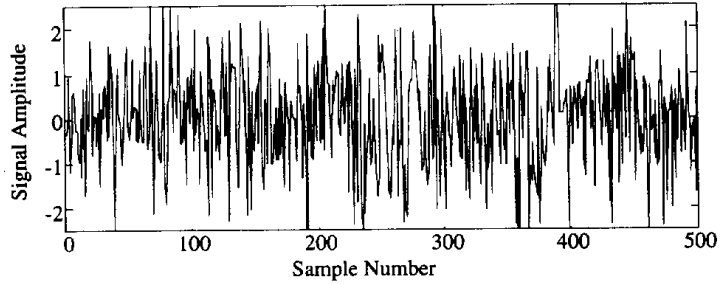

(a)

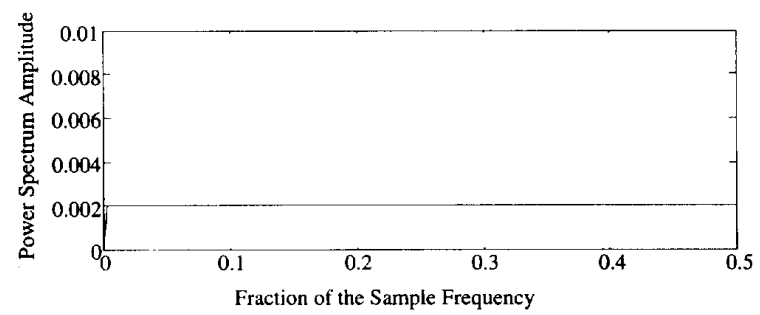

(b)

Fig. 6. Periodic random noise versus fraction of output rate. (a) Time domain data record. (b) Power spectrum of one data record.

\section{The Excitation SignaL}

With the nonlinear features of the induction machine properly considered, input and output data, to which the model will be fitted, must be collected. To ensure that these data represent the dynamic characteristics of the induction machine, the relevant dynamics must be sufficiently excited. Normally, a maximum signal-to-noise ratio is desirable. However, because this technique requires a linear approximation of the machine's behavior about an operating point, too much excitation will cause the nonlinear response to deviate from the linear approximation. Furthermore, what might be a reasonable excitation at one operating point could easily be excessive at another operating point. It is important to consider this compromise when selecting the excitation signal.

A frequently selected excitation signal is the inherent noise from pulse width modulation (PWM). Generally the noise resulting from the PWM does not sufficiently excite the machine's dynamics. This is because the switching frequency of the PWM is considerably faster than the electrical dynamics of the induction machine. It is too fast to excite the mechanical, magnetic, or electrical dynamics. It would indeed be a very poor PWM if, in the normal operation of the induction machine, it disturbed the machines dynamics.

A second possible excitation signal, periodic random noise, is shown in Fig. 6. This signal has a theoretically uniform excitation over a defined range of frequencies, as is shown in its power spectrum of Fig. 6. However, the noise signal has an amplitude range that makes it difficult if not impossible to sufficiently excite the dynamics while not disturbing them so much that the linear approximation about an operating point is invalid. This excitation signal is also not generally appropriate for this system.

Another possible signal, uniform white noise (see Fig. 7) does not as uniformly excite a range of dynamics during any one data record, as is shown by its power spectrum in Fig. 7. However, it does disturb the machine more evenly about an 


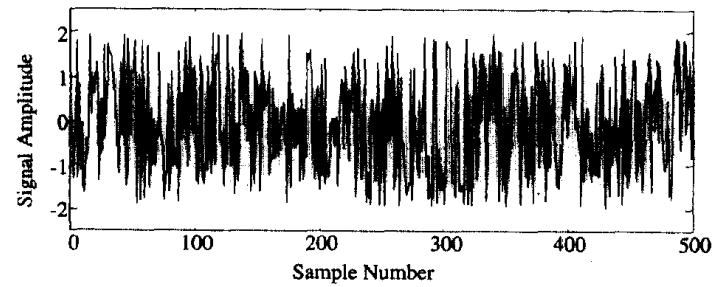

(a)

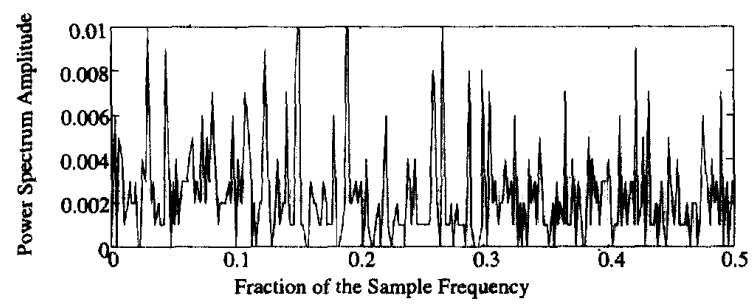

(b)

Fig. 7. Uniform white noise versus fraction of output rate. (a) Time domain data record. (b) Power spectrum of one data record.

operating point. This allows the dynamics to be sufficiently excited but does not cause the machine to deviate too much from the operating point. While this difference between periodic random and uniform white noise may appear subtle from these figures, the effect on the accuracy and consistency of the estimation results is clearly noticeable. Therefore, uniform white noise is chosen as the most appropriate excitation signal.

The sample rate at which the excitation signal is output also affects the excitation of the dynamics. This effect can be used to distinguish between the electrical and magnetic dynamics. By outputting the noise slowly enough that the electrical dynamics are not disturbed, the magnetic dynamics can be better represented in the sampled data. This, in addition to different sample rates, enables the subsystems to be separated and therefore more accurately identified.

\section{THE IDENTIFICATION EXPERIMENT}

To identify the physical parameters of an induction machine, two separate identification experiments were performed. The first identified the stator reflected transient break frequency, resistance, and inductance from the electrical subsystem model. The second identified the rotor break frequency from the model of the magnetic subsystem. This section will describe the equipment used and explain these experiments in detail.

\section{A. Equipment and Analytical Tools Used}

In order to perform time series analysis ( $z$-transform estimation) on a $3 \emptyset, 2$-hp, four-pole field oriented induction machine, noise was injected into the machine from a Macintosh IIx operating a Lab VIEW ${ }^{\mathrm{TM}} 3.0$ virtual instrument which we designed specifically to facilitate a productive user interface for this task. (Note: The Lab View virtual instrument software allows the user to develope a customized instrumentation front panel on the computer screen). The line-to-line voltages and phase currents were scaled such that rated values could be measured with half of the converters' range. The signals were filtered with a first order lowpass filter to avoid alias frequencies, then sampled simultaneously while noise was output. A 12-b $\pm 5 \mathrm{~V}$ analog-to-digital converter was used to sample the signals. The sampled data were then loaded into MATLAB where they were converted to $d q$ synchronous frame values, digitally filtered and resampled at various rates. The coefficients of the discrete time model were found with least squares optimization using the ARMA tools in the system identification toolbox of MATLAB.

\section{B. The Electrical Subsystem}

To identify the stator reflected transient break frequency, the electrical subsystem model (10) was used. The induction machine was operated in field orientation via a digital, indirect vector controller that maintained constant rotor flux independent of operating points. Using a dc machine connected along the same shaft, the induction machine was loaded with the $\mathrm{dc}$ machine running with a closed velocity and position loop (approximately $25-\mathrm{Hz}$ and $5-\mathrm{Hz}$ bandwidths respectively). This forced the speed to remain constant. With the flux and speed held constant, the assumptions used to extract the electrical subsystem model from the operating point model are no longer approximations. Then, in order to identify the specific values for the stator transient resistance and inductance, the static gain was determined using step responses about the operating points.

While the assumptions of constant flux and speed can be made true using the field oriented controller of the induction machine and the velocity controller of the $\mathrm{dc}$ machine, in most applications connecting an additional machine to provide constant speed is not practical. Furthermore, these assumptions are very good approximations in the discrete time domain, where an appropriate sample rate for the electrical dynamics can force the magnetic and mechanical time constants to appear infinite. If the induction machine, then, is operated with only a current regulator, without the flux and speed forced to be constant, the dynamic pole of the electrical subsystem can still be accurately identified.

To estimate the electrical subsystem and demonstrate the suitability of the approximations of constant flux and speed relative to the electrical dynamics, both methods of operating the induction machine were used.

With the induction machine operating with field orientation, uniform white noise was input to the machine as a $q$-axis (torque) command. When the induction machine was operating without the constraints of constant flux and speed, the excitation signal was input as stationary $d$-and $q$-axis current commands. Under both methods of operating, this noise was output at $200 \mathrm{~Hz}$, a rate sufficient to excite the anticipated electrical dynamics.

The accuracy of the identification results was assessed by comparing, for various amplitudes of noise, the standard deviations of the coefficients of both the discrete and continuous time models, the loss and Akaike's final prediction error functions [10], and the consistency of estimated values across a range of moderate sample rates. For the electrical subsystem, sample rates of $200-400 \mathrm{~Hz}$ were used to compare and analyze the estimation results. For the operating points analyzed, a sample rate of $200 \mathrm{~Hz}$ was found to be the most appropriate. 
TABLE 1

Parameter Estimates from Electrical Subsystem

\begin{tabular}{cc}
\hline$\omega_{r}(\mathrm{r} / \mathrm{min})$ & $\frac{r_{s}^{\prime}}{\sigma L_{s}}(\mathrm{rad} / \mathrm{s})$ \\
\hline 750 & 227 \\
1000 & 232 \\
1250 & 239 \\
1500 & 235 \\
1700 & 227 \\
\hline
\end{tabular}

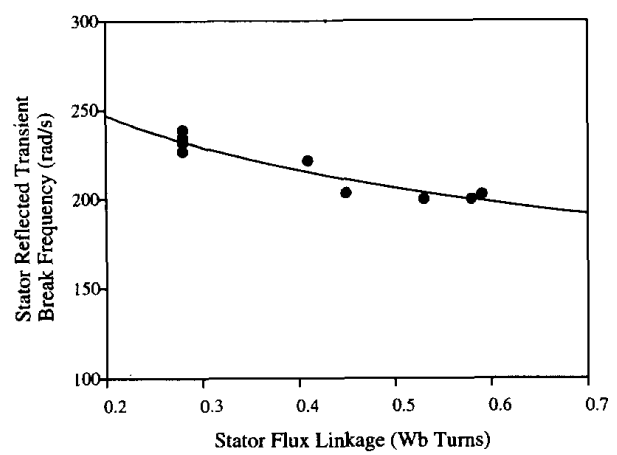

Fig. 8. Estimated stator reflected transient time constant versus flux.

As the flux did not remain constant for various operating points, the stator reflected transient break frequency was evaluated as a function of flux. The stationary frame flux was calculated from the known operating point values for $\overrightarrow{\mathbf{V}}_{q d s o}^{s}, \overrightarrow{\mathbf{I}}_{q s d o}^{s}$, and $\omega_{e}$, and from the previously identified value of $\mathrm{r}_{s}^{\prime}$. The flux was then converted to the synchronous frame.

\section{The Magnetic Subsystem}

The magnetic subsystem model (11) was used to identify the rotor time constant. The induction machine was not run in field orientation because the rotor time constant cannot be identified if the instantaneous flux remains constant. A current regulator was used, however, to prevent excessive currents. The $\mathrm{dc}$ machine, operating with a closed velocity loop, was again connected along the same shaft, forcing the speed to remain constant. This satisfied the assumption of constant speed used to derive the magnetic subsystem model.

Again, in most applications, the addition of a second machine to maintain constant speed is not practical. Therefore, as with the identification experiment to estimate the electrical dynamics, the magnetic dynamics were estimated with and without the $\mathrm{dc}$ machine maintaining constant speed. This will also demonstrate the suitability of the approximation of constant speed relative to the magnetic dynamics.

Uniform white noise was input to the machine as stationary $d$-and $q$-axis current commands. In order to sufficiently excite the magnetic dynamics while not disturbing the electrical dynamics, the noise was output at $33 \mathrm{~Hz}$, exciting all frequencies up to $17 \mathrm{~Hz}$.

The accuracy of the magnetic subsystem identification was assessed similarly to that of the electrical subsystem. For the magnetic subsystem, sample rates in the range of $33-83 \mathrm{~Hz}$ were used to analyze the estimation results. A sample rate of $50 \mathrm{~Hz}$ was found to be the most appropriate for the operating points used.

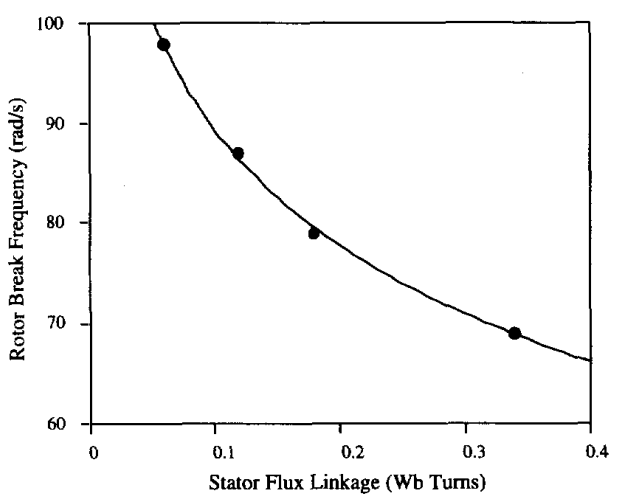

Fig. 9. $\frac{1}{\tau_{r}}$ estimates versus operating point flux (speed held constantat each operating point).

Again, the flux did not remain constant for various operating points. The rotor time constant, therefore, was evaluated as a function of flux. The synchronous frame flux was calculated in the same manner as previously described.

\section{RESULTS}

Experiments were performed to determine both stator transient dynamics and rotor dynamics, i.e., the electrical and magnetic dynamics respectively. Both constant flux, constant speed procedures and varying flux, varying speed procedures were evaluated.

\section{A. Electrical Subsystem (Transient Dynamics) Results}

The stator-reflected transient resistance, transient inductance, and transient break frequency were estimated using the techniques outlined in this paper.

First, with rotor flux and speed held constant, the transient break frequency was estimated at various operating points. The results are shown in Table I. From these break frequencies and the static gain estimated at these operating points, the stator reflected transient resistance and inductance were estimated to be $2.1 \Omega$ and $9.1 \mathrm{mH}$, respectively. The consistency of the estimated parameters at various operating points is expected given the constant flux provided by the field oriented controller.

Next, the constraints of constant speed and flux were removed and the identification process was performed as described previously in this paper. The estimated stator transient break frequency versus synchronous frame operating point ( $d$-axis) flux is shown in Fig. 8.

\section{B. Magnetic Subsystem (Rotor Dynamics) Results}

The rotor break frequency, $\frac{1}{\tau_{r}}$, was evaluated at various operating points. Since the operating point flux was allowed to vary, Figs. 9 and 10 map the estimated value for $\frac{1}{\tau_{r}}$ as a function of the synchronous frame operating point ( $d$-axis) flux. Fig. 9 shows the rotor break frequency estimated while speed was maintained at a constant value for each operating point. Fig. 10 shows the estimated values for $\frac{1}{\tau_{r}}$ with and without the constraint of constant velocity. 


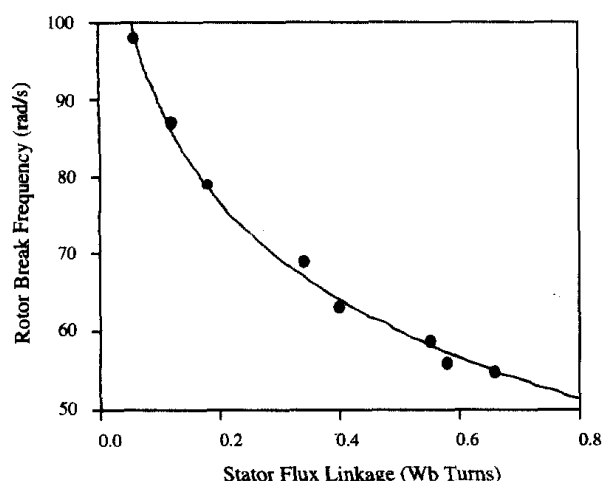

Fig. 10. $\frac{1}{\tau_{r}}$ estimates versus operating point flux (speed allowed to vary).

The smooth trends and realistic values of the results of both the electrical and magnetic identification experiments indicate that the assumptions of constant speed and flux are valid for this parameter estimation method if an appropriate sample rate is selected.

\section{CONCLUSION}

This paper has presented an easily implemented selfcommissioning approach capable of achieving accurate physical parameter estimation in ac machines, especially induction machines. The approach overcomes the limitations of previous approaches by providing:

- A correct operating point model whose physical parameters vary with operating space.

- Multi-rate sampling methods which optimize accuracy for transient electrical, magnetizing, and mechanical dynamics, given known or preselected A/D converter resolutions.

- Excitation methods to optimally use existing converters for improved estimation accuracy.

Using the techniques outlined in this paper, more accurate physical parameter estimates can be obtained for any ac machine system, including those with widely separated time constants, those with varying physical parameters, and those with continuous nonlinear features.

\section{REFERENCES}

[1] G. E. P. Box and G. M. Jenkins, Time Series Analysis, Forecasting and Control. San Francisco, CA: Holden Day, 1970.

2] J. S. Bendat and A. G. Piersol, Random Data: Analysis and Measurement Procedures. New York: Wiley-Interscience, 1971.

[3] L. Ljung, System Identification: Theory For The User. Englewood Cliffs, NJ: Prentice Hall, 1987.

[4] J. Holtz and T. Thimm, "Identification of machine parameters in a vector controlled induction motor drive," in Proc. 1989 IEEE Ind. Applicat. Soc. Annu. Conf., pp. 601-606.

[5] M. Vélez-Reyes, K. Minami, and G. C. Verghese, "Recursive speed and parameter estimation for induction machines," in Proc. 1989 IEEE Ind. Applicat. Soc. Conf. pp. 607-611.

[6] J. Stephan, M. Bodson, and J. Chiasson, "Real-time estimation of the parameters and fluxes of induction motors," in Proc. 1992 IEEE Ind. Applicat. Soc. Conf., pp. 578-585.

[7] G. Fantauzzi, "The relation between the sampling time and stochastic error for the impulsive response of linear time-independent systems," IEEE Trans. Automat. Contr., vol. AC-13, pp 426-428, 1968.
[8] R. D. Lorenz, T. A. Lipo, and D. W. Novotny, "Motion control with induction motors" to be published in IEEE Proc. 1994.

[9] K. Minami, M. Vélez-Reyes, D. Elten, G. Verghese, and D. Filbert, "Multi-stage speed and parameter estimation for induction machines," in Proc. 1991 IELEE Power Electron. Specialisss Conf., pp. 596-604.

[10] G. E. P. Box, G. M. Jenkins, and G. C. Reinsel, Time Series Analysis, Forecasting and Control, 3rd ed. Englewood Cliffs, NJ: Prentice Hall, 1994

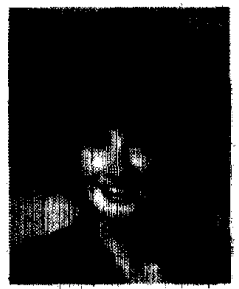

Diane E. Borgard received the B.S. degree in interdisciplinary engineering from Purdue University West Lafayette, IN, in 1992, and the M.S. degree in electrical and computer engineering from the University of Wisconsin, Madison, in 1994.

She worked as a Research Assistant at the University of Wisconsin, under Dr. Lorenz, for the Wisconsin Electric Machines and Power Electronics Consortium. During this time, she engaged in a study of machine control and parameter estimation. She currently works at home in St. Charles, MO.

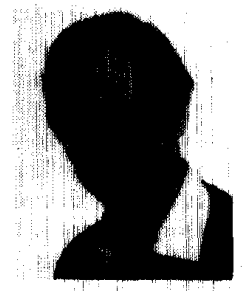

Gustaf Olsson received his engineering education and the licentiat degree in autamatic control at the Royal Institute of Technology, Stockholm, Sweden.

He worked at the Swedish State Power Board for two years before he was appointed Assistant Professor in Automatic Control at the Lund Institute of Technology, Sweden, in 1967. In 1970, he became Associate Professor and Chairman of the Automatic Control Department. In 1987, he was appointed Professor and Chair in Industrial Automation. He is presently head of the Department of Industrial Electrical Engineering and Automation. He has been a visiting scientist or professor at the University of California, Los Angeles, University of Houston, TX, Systems Control, Inc. Palo Alto, CA, and the University of Wisconsin, Madison, respectively. He has held shorter research positions at Kyoto Univerity, Japan, and the University of Queensland, Brisbane, Australia. He is presently an international advisor for research groups at the University of Queensland. His main research interests are modeling, estimation, and contro of industrial processes. He has written about 65 publications, one international textbook, and contributed to four other books.

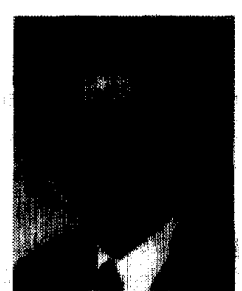

Robert D. Lorenz (S'83-M'84-SM'91) received the B.S., M.S., and Ph.D. degrees from the University of Wisconsin, Madison, in 1969, 1970, and 1984, respectively. He did his M.S. thesis research at the Technical University of Aachen, West Germany from 1969 to 1970.

From 1972 to 1982 , he was a member of the research staff at the Gleason Works, Rochester, NY. Since 1984, he has been a member of the faculty of the University of Wisconsin, Madison, where he is Professor of mechanical engineering and of electrical and computer engineering. In this position, he acts as Associate Director of the Wisconsin Electric Machines and Power Electronics Consortium and as Co-Director of the Advanced Automation and Robotics Consortium. He was a Visiting Research Professor in the Electrical Drives Group, Catholic University of Leuven, Belgium, and at the Electrical Drives Institute, Technical University of Aachen, in the summers of 1989 and the summers of 1987 and 1991, respectively. He is an active consultant to many organizations. His current research interests include sensor integrated electromagnetic actuator technologies, real time digital signal processing and estimation techniques, and ac drive and high precision machine control technologies.

Dr. Lorenz is a past Chairman of the IEEE Industry Applications Society Industrial Drives Committee, the Electrical Machines Committee, and the Industrial Power Converter Committee. He is a Registered Professional Engineer in the states of New York and Wisconsin. He is a member of ASME, ISA, and SPIE. 